\title{
Activation and Conjugation of Soluble Polysaccharides using 1-Cyano-4-Dimethylaminopyridine Tetrafluoroborate (CDAP)
}

\author{
Andrew Lees ${ }^{1}$, James Zhou $^{1}$ \\ ${ }^{1}$ Fina Biosolutions LLC
}

\section{Corresponding Author}

Andrew Lees

alees@finabio.com

\section{Citation}

Lees, A., Zhou, J. Activation and

Conjugation of Soluble Polysaccharides using 1-Cyano-4-Dimethylaminopyridine Tetrafluoroborate (CDAP). J. Vis. Exp.

(172), e62597, doi:10.3791/62597 (2021).

\section{Date Published}

June 14, 2021

DOI

$10.3791 / 62597$

URL

jove.com/video/62597

\section{Abstract}

Conjugate vaccines are remarkable advances in vaccinology. For the preparation of polysaccharide conjugate vaccines, the polysaccharides can be conveniently functionalized and linked to vaccine carrier proteins using 1-cyano-4dimethylaminopyridine tetrafluoroborate (CDAP), an easy-to-handle cyanylating reagent. CDAP activates polysaccharides by reacting with carbohydrate hydroxyl groups at $\mathrm{pH} 7-9$. The stability and reactivity of CDAP are highly $\mathrm{pH}$-dependent. The $\mathrm{pH}$ of the reaction also decreases during activation due to the hydrolysis of CDAP, which makes good $\mathrm{pH}$ control the key to reproducible activation. The original CDAP activation protocol was performed at room temperature in unbuffered $\mathrm{pH} 9$ solutions.

Due to the rapid reaction under this condition ( $<3 \mathrm{~min}$ ) and the accompanying fast $\mathrm{pH}$ drop from the rapid CDAP hydrolysis, it was challenging to quickly adjust and maintain the target reaction $\mathrm{pH}$ in the short time frame. The improved protocol described here is performed at $0{ }^{\circ} \mathrm{C}$, which slows CDAP hydrolysis and extends the activation time from $3 \mathrm{~min}$ to $\sim 15 \mathrm{~min}$. Dimethylaminopyridine (DMAP) was also used as a buffer to pre-adjust the polysaccharide solution to the target activation $\mathrm{pH}$ before adding the CDAP reagent. The longer reaction time, coupled with the slower CDAP hydrolysis and the use of DMAP buffer, makes it easier to maintain the activation $\mathrm{pH}$ for the entire duration of the activation process. The improved protocol makes the activation process less frenetic, more reproducible, and more amenable to scaling up.

\section{Introduction}

Conjugate vaccines, such as those consisting of polysaccharides covalently linked to a carrier protein, are among the remarkable advances in vaccinology 1,2 .
Polysaccharides, as T-cell-independent antigens, are poorly immunogenic in infants and do not induce memory, class switching, or affinity maturation of antibodies ${ }^{3}$. These 
shortcomings are overcome in polysaccharide conjugate vaccines ${ }^{4}$. As most polysaccharides do not have a convenient chemical handle for conjugation, they must first be made reactive or "activated." The activated polysaccharide is then linked either directly with the protein (or modified protein) or is functionalized for additional derivatization before conjugation ${ }^{4}$. Most licensed polysaccharide conjugate vaccines use either reductive amination or cyanylation to activate polysaccharide hydroxyls. Cyanogen bromide $(\mathrm{CNBr})$, a reagent that had previously been used to activate chromatography resins, was initially used for polysaccharide derivatization. However, $\mathrm{CNBr}$ requires high $\mathrm{pH}$, typically $\mathrm{pH} 10.5$ or greater, to partially deprotonate polysaccharide hydroxyls so that they are sufficiently nucleophilic to attack the cyano group. The high $\mathrm{pH}$ can be detrimental to base-labile polysaccharides, and neither CNBr nor the active cyano-ester initially formed is sufficiently stable at such high $\mathrm{pH}$.

CDAP (1-cyano-4-dimethylaminopyridine tetrafluoroborate; Figure 1) was introduced by Lees et al. for use as a cyanylating agent for the activation of polysaccharides ${ }^{5,6}$. CDAP, which is crystalline and easy to handle, was found to activate polysaccharides at a lower $\mathrm{pH}$ than $\mathrm{CNBr}$ and with fewer side reactions. Unlike CNBr, CDAPactivated polysaccharides can be directly conjugated to proteins, simplifying the synthesis process. CDAPactivated polysaccharides can be functionalized with a diamine (e.g., hexane diamine) or a dihydrazide (e.g., adipic dihydrazide, $\mathrm{ADH}$ ) to make amino- or hydrazidederivatized polysaccharides. A high concentration of the homobifunctional reagent is used to suppress crosslinking of polysaccharides. Amino polysaccharides can then be conjugated using any of the myriad techniques used for protein conjugation. Hydrazide-derivatized polysaccharides are often coupled to proteins using a carbodiimide reagent (e.g., 1-ethyl-3-(3-dimethylaminopropyl)carbodiimide $(E D A C))^{7}$. Further optimization of CDAP polysaccharide activation has been described by Lees et al. ${ }^{8}$ and is incorporated into the protocol described here.

\section{CDAP conjugation overview}

The CDAP protocol can be conceptualized as two phases:

(1) the activation of the polysaccharide and (2) conjugation of the activated polysaccharide with a protein or ligand (Figure 2). The goal of the first step is to efficiently activate the polysaccharide, while the goal of the second is to efficiently conjugate to the activated polysaccharide. The activated polysaccharide ties the two steps together. This conceptualization helps focus on the critical elements of each step. Figure 2 expands on this conceptualization, showing the desired activation and coupling reactions, along with the hydrolysis reactions and side reactions.

During the activation phase, the three major concerns are CDAP stability, CDAP reaction with the polysaccharide hydroxyls, and the stability of the activated polysaccharide (Figure 3). CDAP hydrolysis increases with $\mathrm{pH}$, as does the hydrolysis of the activated polysaccharide and the side reactions. However, the CDAP reaction with the polysaccharide is facilitated by increasing the $\mathrm{pH}$. Efficiently activating polysaccharides with CDAP requires a balance between 1) the reactivity of the polysaccharide and CDAP and 2) the hydrolysis and side reactions of both the reagent and the activated polysaccharide.

In the original CDAP activation protocol described by Lees et al. ${ }^{5}$, CDAP activation of polysaccharides was carried out at room temperature in unbuffered $\mathrm{pH} 9$ solution. The rate of activation was found to be rapid under this condition, and the activation would be complete within $3 \mathrm{~min}$. The reaction was also accompanied by rapid hydrolysis of CDAP, causing 
a rapid $\mathrm{pH}$ drop of the unbuffered reaction solution. It was challenging to quickly raise and to maintain the reaction $\mathrm{pH}$ at the target value in such a short time frame. In the described protocol, activation was performed by adding CDAP from a $100 \mathrm{mg} / \mathrm{mL}$ stock solution to the unbuffered polysaccharide solution. The $\mathrm{pH}$ was raised $30 \mathrm{~s}$ later with "an equal volume of $0.2 \mathrm{M}$ triethylamine". Protein to be conjugated was then added after $2.5 \mathrm{~min}$ to the activation reaction. Notably, the $\mathrm{pH}$ of the activation step was not well controlled and most likely initially exceeded the target $\mathrm{pH}$. The fast reaction requiring prompt $\mathrm{pH}$ adjustment made the activation process difficult to control and challenging to scale up.

In contrast to the original protocol, the modified protocol described here has two major improvements. First, the $\mathrm{pH}$ of the polysaccharide solution is pre-adjusted to target activation $\mathrm{pH}$, using DMAP as the buffer, before the addition of CDAP. DMAP has a pKa of 9.5 and thus has good buffering power around $\mathrm{pH} \mathrm{9}$, and unlike many other buffers, DMAP was not found to promote CDAP hydrolysis ${ }^{8}$. Furthermore, DMAP is already a process intermediate and therefore does not add a new component to the reaction mixture. Pre-adjusting the $\mathrm{pH}$ before adding CDAP eliminates the large $\mathrm{pH}$ swing at the beginning of the reaction and allows for more efficient maintenance of the target $\mathrm{pH}$ during the reaction. The second improvement is to perform the activation reaction at $0{ }^{\circ} \mathrm{C}$, where the rate of CDAP hydrolysis is markedly slower than that at room temperature. With the longer reagent halflife at $0{ }^{\circ} \mathrm{C}$, the activation time is increased from $3 \mathrm{~min}$ to $15 \mathrm{~min}$ to compensate for the slower activation rate at the lower temperature. The longer reaction time, in turn, makes it easier to maintain the reaction $\mathrm{pH}$. The use of $0{ }^{\circ} \mathrm{C}$ also slows the degradation of $\mathrm{pH}$-sensitive polysaccharides, making it possible to prepare conjugates of this type of polysaccharide. The improvements in the protocol make the activation process less frenetic, easier to control, more reproducible, and more amenable to scaling up.

This article describes the improved protocol for carrying out controlled CDAP activation of polysaccharide at $0{ }^{\circ} \mathrm{C}$ and at a specified target $\mathrm{pH}$ and performing subsequent derivatization of the activated polysaccharides with ADH. Also described is a trinitrobenzene sulfonic acid (TNBS) assay, based on the method of Qi et al. ${ }^{9}$, for the determination of hydrazide level on the modified polysaccharide. A modified assay for hexoses based on resorcinol and sulfuric acid ${ }^{10}$ is also described, which can be used for determining a broader range of polysaccharides. For more information on CDAP activation and conjugation, the reader is referred to earlier publications $5,6,8$ by Lees et al.

\section{Protocol}

NOTE: Prepare the polysaccharide solution, ADH solution, DMAP solution, and CDAP stock solution in advance before executing the polysaccharide activation and functionalization procedures. Place the solutions and equipment in an organized, convenient, and logical location. The reaction described is for $10 \mathrm{mg}$ of polysaccharide and can be scaled up or down. It is recommended to evaluate the protocol at a small scale before scaling up.

\section{Prepare $5 \mathrm{mg} / \mathrm{mL}$ polysaccharide solution, 2 $\mathrm{mL}$.}

1. For lyophilized polysaccharide

1. Allow the polysaccharide container to come to room temperature before opening. Weigh out $10 \mathrm{mg}$ of polysaccharide inside a screw-cap tube using an analytical balance. Use a static eliminator for 
easier sampling and more accurate weighing of the powder.

2. Add $2 \mathrm{~mL}$ of $0.15 \mathrm{M}$ sodium chloride $(\mathrm{NaCl})$ to the tube to dissolve the polysaccharide. Cap and vortex the tube.

NOTE: Sodium chloride does not affect the CDAP reaction, but it may affect the polysaccharide secondary structure. Some polysaccharides are more soluble at different salt concentrations.

3. Mix the tube by end-over-end rotation for $12-24$ $\mathrm{h}$, depending on the polysaccharide molecular weight, to allow the polysaccharide to hydrate fully. If necessary, gently warm the tube to promote solubilization.

2. For solubilized polysaccharide in buffered solution NOTE: For efficient CDAP activation, the polysaccharide solution should not contain any buffer, especially phosphate ion. Follow the procedure below to replace the buffer with water or a saline solution and to adjust the polysaccharide concentration to $5 \mathrm{mg} / \mathrm{mL}$.

1. Obtain a $4 \mathrm{~mL}$ or $15 \mathrm{~mL}$ centrifugal-spin filter device of the appropriate molecular weight cutoff (MWCO). NOTE: The MWCO is ideally 5-10 times smaller than the molecular weight of the polysaccharide.

2. Add a volume of the buffered polysaccharide solution containing $\sim 20 \mathrm{mg}$ of the polysaccharide to the filter insert. Fill to the full mark with water or a saline solution. Cap the filter tightly. Mix by end-overend a few times.

3. Centrifuge the filter device at the centrifugal force suggested by the manufacturer. Ensure that the centrifugation time is long enough to achieve at least
5 -fold volume reduction after each spin. Discard the flow-through. Reassemble the filter device.

4. Refill the filter insert to the full mark with fresh water or saline solution. Cap the filter tightly. Mix the content in the filter by end-over-end rotation $\sim 10$ times or by gentle vortexing; repeat the spin.

NOTE: Polysaccharide can accumulate at the bottom of the filter insert of the centrifugal device, forming a gel. It is recommended to re-mix the retentant inside the filter insert with the fresh refill before the next spin.

5. Repeat the refill and spin cycle for a minimum of 3 times.

6. Follow the exercise below to recover the polysaccharide retentant from the filter insert.

1. Add fresh water or saline to the filter insert so that the volume is $\sim 1 \mathrm{~mL}$. Mix by pipetting up and down or by gentle vortexing.

2. Transfer all of the mixed retentant to a $5 \mathrm{~mL}$ tube. Add $1 \mathrm{~mL}$ of fresh water or saline to the filter insert. Rinse the filter by pipetting up and down or by gentle vortexing. Transfer and combine all of the rinses with the recovered polysaccharide.

7. Determine the polysaccharide concentration (see the polysaccharide assay in section 7.3). Dilute the polysaccharide with additional water or saline to 5 $\mathrm{mg} / \mathrm{mL}$.

3. When the polysaccharide solution is prepared, chill the tube containing the polysaccharide solution in an ice bucket. 


\section{Prepare $0.5 \mathrm{M}$ adipic acid dihydrazide (ADH) solution, $10 \mathrm{~mL}$.}

1. Weigh $0.87 \mathrm{~g} \mathrm{ADH}$ in an analytical balance, and solubilize in $8 \mathrm{~mL}$ of $0.1 \mathrm{M}$ HEPES (4-(2-hydroxyethyl)-1piperazineethanesulfonic acid), $\mathrm{pH} 8$.

2. Adjust to the target $\mathrm{pH}$ with $1 \mathrm{M}$ sodium hydroxide $(\mathrm{NaOH})$, monitored by a $\mathrm{pH}$ meter. Bring to $10 \mathrm{~mL}$ with additional buffer and re-confirm the $\mathrm{pH}$.

\section{Prepare 2.5 M DMAP solution, $10 \mathrm{~mL}$.}

NOTE: DMAP is toxic and will penetrate the skin. Wear nitrile gloves when performing the procedure.

1. Carefully weigh $3 \mathrm{~g}$ of DMAP into a $50 \mathrm{~mL}$ conical tube. Add $5 \mathrm{ml}$ of water to DMAP and mix by vortexing for 5 min to obtain a cloudy solution $(\sim 7 \mathrm{~mL})$.

2. While mixing, add $50 \mu \mathrm{L}$ increments of $10 \mathrm{~N}$ hydrochloric acid $(\mathrm{HCl})$ to the DMAP solution. Mix between each addition. Stop adding when the solution becomes clear.

3. Add $10 \mathrm{~N} \mathrm{NaOH}$ in $25 \mu \mathrm{L}$ increments to bring the DMAP solution to $\sim \mathrm{pH} 8$.

4. Bring the DMAP solution to $10 \mathrm{~mL}$ with water to give a $2.5 \mathrm{M}$ solution.

5. Fine-tune the $\mathrm{pH}$ of the $2.5 \mathrm{M}$ DMAP solution.

NOTE: DMAP solution $\mathrm{pH}$ changes with concentration and ionic strength. This exercise is to fine-tune the 2.5 M DMAP stock to a specific $\mathrm{pH}$ so that when it is mixed with 10 volumes of polysaccharide, the resulting solution is close to the target $\mathrm{pH}$ for activation.

1. Prepare a series of $1.5 \mathrm{~mL}$ tubes containing $1 \mathrm{~mL}$ of water or the $\mathrm{NaCl}$ solution, whichever was used for preparing the polysaccharide solution. Chill the tubes on ice.

2. Add $100 \mu \mathrm{L}$ of DMAP to a chilled tube. Vortex and measure the $\mathrm{pH}$ with a $\mathrm{pH}$ meter. Then, discard the measured tube.

3. If the measured $\mathrm{pH}$ is not close to the target value, adjust the $\mathrm{pH}$ of the DMAP stock with $1 \mathrm{M} \mathrm{NaOH}$ or $\mathrm{HCl}$ as appropriate. Repeat steps 3.5.2 and 3.5.3 until the measured $\mathrm{pH}$ is close to the target $\mathrm{pH}$.

\section{Prepare $100 \mathrm{mg} / \mathrm{mL}$ CDAP stock solution}

NOTE: CDAP powder should be kept tightly closed and stored at $-20^{\circ} \mathrm{C}$ and allowed to come to room temperature before opening. Wear nitrile gloves when performing the procedure.

1. Tare a $1.5 \mathrm{~mL}$ snap-cap microcentrifuge tube on an analytical balance. Using a small spatula, weigh out $10-140 \mathrm{mg}$ of CDAP into the tube. Note the actual weight of CDAP.

2. Determine the volume of acetonitrile needed to prepare $100 \mathrm{mg} / \mathrm{mL}$ CDAP. Open acetonitrile in a fume hood.

3. Using an appropriate volume pipet, draw and release the acetonitrile to equilibrate its vapor in the pipet tip. Wait for the solvent to drip out of the pipet tip after a few seconds, and be prepared to transfer it to the CDAP tube directly. Draw the calculated volume of acetonitrile and directly transfer it to the CDAP tube. Snap the cap shut.

NOTE: Acetonitrile can also be transferred to the CDAP tube using a Hamilton syringe or its equivalent of suitable size.

4. Vortex to fully solubilize the CDAP. Place the CDAP tube in an ice bucket. 
NOTE: CDAP is stable in acetonitrile in the cold. Soluble stocks may be kept at $-20^{\circ} \mathrm{C}$ for $>1$ week. However, it is preferable to prepare fresh CDAP solutions.

\section{Polysaccharide activation and hydrazide functionalization}

1. Ensure all of the following items are ready and solutions chilled on ice before starting the activation: $2 \mathrm{~mL}$ of a $5 \mathrm{mg} / \mathrm{mL}$ polysaccharide solution in a flat-bottom, widemouth container containing a stir bar, placed on top of a magnetic stirrer; $100 \mathrm{mg} / \mathrm{mL}$ CDAP stock solution; $2.5 \mathrm{M}$ DMAP stock solution; a $\mathrm{pH}$ meter with a semi-micro $\mathrm{pH}$ probe, such as the $6 \mathrm{~mm}$ diameter probe, calibrated for $0{ }^{\circ} \mathrm{C}$ according to the manufacturer's instructions; a 100 $\mu \mathrm{L}$ pipet ready to use; a timer cleared and ready to use; an autotitrator dispenser head positioned or $10 \mu \mathrm{L}$ pipet ready to use; $0.5 \mathrm{M} A D H$ solution.

2. Pre-adjust the $\mathrm{pH}$ of the polysaccharide to the target $\mathrm{pH}$ using DMAP.

1. Place the $\mathrm{pH}$ probe into the polysaccharide solution and leave it in the solution during the entire activation procedure.

2. Transfer $200 \mu \mathrm{L}$ of the DMAP stock solution to the polysaccharide solution by dropwise addition under stirring.Adjust the $\mathrm{pH}$ of the solution to the target activation $\mathrm{pH}$. Add $0.1 \mathrm{M} \mathrm{HCl}$ to lower the $\mathrm{pH}$ and $0.1 \mathrm{M} \mathrm{NaOH}$ to increase the $\mathrm{pH}$. Avoid exceeding the target $\mathrm{pH}$ by more than $0.1 \mathrm{pH}$ unit, and keep the reaction chilled in an ice-water bath for the duration of the activation.

3. CDAP activation
1. Pipet $100 \mu \mathrm{L}$ CDAP up and down to equilibrate the vapor in the pipet tip. Transfer $100 \mu \mathrm{L}$ of CDAP to the polysaccharide solution with stirring.

NOTE: This activation uses $1 \mathrm{mg}$ of CDAP for 1 $\mathrm{mg}$ of polysaccharide as a starting ratio. The ratio can be increased or decreased when optimizing the activation.

2. Start the timer and monitor the $\mathrm{pH}$ change during the entire activation. Maintain the reaction at the target $\mathrm{pH}$ by promptly adding $10 \mu \mathrm{L}$ increments of $0.1 \mathrm{M}$ $\mathrm{NaOH}$ to the reaction, with the aid of an autotitrator dispenser (or pipet).

NOTE: It may help reduce the $\mathrm{pH}$ response time to stir with a $\mathrm{pH}$ probe gently. The $\mathrm{pH}$ drops more rapidly in the beginning, and it may be necessary to add the $0.1 \mathrm{M} \mathrm{NaOH}$ more frequently. As the reaction proceeds, the $\mathrm{pH}$ decrease becomes slower, and the addition becomes less frequent. The $\mathrm{pH}$ should remain essentially unchanged when approaching the optimal activation time, which is 10-15 min for $\mathrm{pH} 9$ activation.

4. $\mathrm{ADH}$ functionalization

1. When the optimal activation time is reached, add $2 \mathrm{~mL}$ of $0.5 \mathrm{M} \mathrm{ADH}$ all at once to the activated polysaccharide under stirring. Check that the $\mathrm{pH}$ is in the target range ( $\mathrm{pH}$ 8-9 for $\mathrm{ADH})$.

NOTE: One addition with quick mixing minimizes the probability for both ends of the dihydrazide to react with the activated polysaccharide, preventing polysaccharide crosslinking.

2. Continue to stir the reaction mixture for at least $1 \mathrm{~h}$. Transfer the reaction mixture to $4{ }^{\circ} \mathrm{C}$, but $0-20^{\circ} \mathrm{C}$ is acceptable. 
NOTE: The ADH functionalization reaction is not strongly dependent on temperature. As the large excess of dihydrazide acts as the quenching reagent, it is not necessary to further quench the activated polysaccharide. However, when directly conjugating proteins, the reaction should be quenched, typically with $1 \mathrm{M}$ glycine, $\mathrm{pH}$ 8-9.

\section{Purification of ADH-functionalized polysaccharide by dialysis}

NOTE: The crude product from the ADH functionalization reaction contains a high concentration of $\operatorname{ADH}(0.5 \mathrm{M})$, which can be removed most efficiently by extensive dialysis. Gel filtration, either with a column or a spin desalting device, is not as efficient, especially when it is required to remove the residual $A D H$ contaminant.

1. Determine the MWCO of the dialysis membrane. Use a $3 \mathrm{kDa}$ cutoff for smaller polysaccharides.

NOTE: The MWCO of the dialysis membrane is ideally 5-10 times smaller than the MW of polysaccharide.

2. Choose the desired dialysis format (cassettes or tubings) and the correct device capacity. Ensure that the device capacity is 2 times larger than the sample volume. Consult the manufactures' instructions for using the device.

3. Hydrate the dialysis membrane in water before use. Transfer the crude derivatized polysaccharide solution to the dialysis device according to manufacturers' instructions.

NOTE: Wear nitrile gloves to avoid contact with DMAP.

4. Dialyze in a container filled with $2-4 \mathrm{~L}$ of $1 \mathrm{M} \mathrm{NaCl}$ and a stir bar. Place the container on a stir plate in a cold room or inside a refrigerator. Stir the dialysate gently and continuously during dialysis.

5. After dialyzing for at least $4 \mathrm{~h}$, change to fresh $1 \mathrm{M} \mathrm{NaCl}$, and dialyze for at least $12 \mathrm{~h}$. Dialyze against 2 changes of $0.15 \mathrm{M}$ saline, each for at least $12 \mathrm{~h}$. If desired, dialyze against 2 changes of water.

6. Verify if all the ADH is removed by testing the overnight dialysate using a quick TNBS test.

1. Obtain 3 borosilicate tubes, label them as negative control (ctrl), positive ctrl, and sample, respectively.

2. To the negative ctrl tube, add $975 \mu \mathrm{L}$ of $0.1 \mathrm{M}$ borate, $\mathrm{pH} 9$.

3. To the positive ctrl tube, add $100 \mu \mathrm{L}$ of $0.05 \mathrm{mM} \mathrm{ADH}$ (0.1 mM hydrazide) and $875 \mu \mathrm{L}$ of $0.1 \mathrm{M}$ borate, $\mathrm{pH}$ 9.

4. To the sample tube, add $500 \mu \mathrm{L}$ of the overnight dialysate and $475 \mu \mathrm{L}$ of $0.1 \mathrm{M}$ borate, $\mathrm{pH} 9$.

5. Add $25 \mu \mathrm{L}$ of $1 \%$ TNBS to all three tubes. Mix well. Place in the dark for $1 \mathrm{~h}$.

6. Compare the color intensity of the 3 tubes in $1 \mathrm{~h}$. Ensure that the sample tube color intensity is inbetween that of the positive ctrl and the negative ctrl, which indicates that the ADH contaminant is down to $0.01 \mathrm{mM}$ or below. Dialyze one more time.

NOTE: It is prudent to reduce the level of the ADH contaminant as much as possible so that the ADH hydrazide accounts for less than $1 \%$ of the total hydrazide in the purified hydrazide-polysaccharide.

7. Recover the derivatized polysaccharide from dialysis. Determine the concentration of the polysaccharides and hydrazide. Calculate the hydrazide/polysaccharide ratio (see section 7 ). If the 
dialyzed polysaccharide must be concentrated to $5-10 \mathrm{mg} / \mathrm{mL}$, consult section 1.2 .

\section{Analysis of hydrazide-derivatized polysaccharides}

NOTE: The purpose of the analysis described here is to determine the polysaccharide concentration, the hydrazide concentration, and the level of hydrazide derivatization in terms of the hydrazide/polysaccharide ratio.

1. Sample preparation

NOTE: Polysaccharides to be assayed need to be free of low molecular weight carbohydrate, amine, or hydrazide impurities. Lyophilized samples should be dry and saltfree to ensure accurate weight measurement. Usually, 1 $\mathrm{mL}$ of a $1-2 \mathrm{mg} / \mathrm{mL}$ solution is adequate for assays.

1. Weigh at least $10 \mathrm{mg}$ of the lyophilized polysaccharide sample on an analytical balance, using a non-static spatula or a static eliminator. Dissolve the polysaccharide in water or saline to a concentration (e.g., $2 \mathrm{mg} / \mathrm{mL}$ ) so that the assay signals fall within the linear range of the standard curve.

2. Mix end-over-end and allow enough time for the sample to dissolve completely. Perform overnight hydration depending on polysaccharide molecular weight.

2. Polysaccharide assay: resorcinol/sulfuric acid method NOTE: The appropriate assay for polysaccharides will depend on the carbohydrate composition of the polymers. The original resorcinol/sulfuric acid assay was intended for hexose sugars ${ }^{10}$. The assay was modified here by raising the temperature of the heating step from $90{ }^{\circ} \mathrm{C}$ to $140{ }^{\circ} \mathrm{C}$. At this higher temperature, the assay loses some specificity but can be used to assay many sugars. However, it is still necessary to determine the suitability of the assay for a particular polysaccharide. Triplicates are recommended for each point, but some accommodation may be necessary due to the capacity of the heating block.

1. Prepare $75 \%$ sulfuric acid

NOTE: Concentrated sulfuric acid is extremely corrosive and can cause severe burns. Perform this procedure in a chemical fume hood. Always pour concentrated acid into water, not vice versa!

1. Add $50 \mathrm{~mL}$ of water to a $200 \mathrm{~mL}$ glass bottle. Place the bottle in a cold water bath. Slowly add $150 \mathrm{~mL}$ of sulfuric acid. Cap the bottle so that it is vented.

2. Allow the solution to equilibrate to room temperature. Use the solution within 3 months.

2. Prepare carbohydrate standards

1. Prepare the unmodified polysaccharide solution at $1 \mathrm{mg} / \mathrm{mL}$ to be used as the standard. Alternatively, use a mix of individual sugars in the ratio found in the repeat unit of the polysaccharide, at $1 \mathrm{mg} / \mathrm{mL}$ of the total sugar concentration, as the standard.

NOTE: Although the sugar mix will usually give the same result as the carbohydrate polymer of identical sugar composition, this should be confirmed experimentally.

3. Ensure that the heating block with tube holders for $13 \times 100$ borosilicate test tubes is functioning. Use a protective pad underneath and surrounding the heating block in case of acid spills. Pre-heat the heating block to $140{ }^{\circ} \mathrm{C}$ for a minimum of $1 \mathrm{~h}$ to 
achieve stable, uniform temperature through all the blocks utilized.

4. Label $13 \times 100$ borosilicate test tubes, triplicate for each standard and each sample. Add 0, 2.5, 5, 7.5, $10 \mu \mathrm{g}$ (or $\mu \mathrm{L}$ ) of the $1 \mathrm{mg} / \mathrm{mL}$ carbohydrate standard to the correspondingly labeled standard tubes. Add water to each tube to bring the volume to $100 \mu \mathrm{L}$. NOTE: The color generated is dependent on specific sugars. As some sugars require more mass to generate the full absorbance range, the actual amounts used for the standard curve may vary.

5. Set up sample assays by adding a volume containing $\sim 5 \mu \mathrm{g}$ of the derivatized polysaccharide to three sample tubes and bring the total volume to 100 $\mu \mathrm{L}$ with water. Alternatively, if the polysaccharide concentration in the sample is unknown, perform a series of 4 -fold dilutions. Test $100 \mu \mathrm{L}$ of each dilution in triplicate.

6. Prepare fresh resorcinol at $6 \mathrm{mg} / \mathrm{mL}$ in deionized (dl) water immediately before use. Vortex until the resorcinol is in solution. Add $100 \mu \mathrm{L}$ of $6 \mathrm{mg} / \mathrm{mL}$ resorcinol to each tube.

7. Carefully pour the estimated amount of $75 \%$ sulfuric acid into a small beaker.

NOTE: Wear a lab coat, nitrile gloves, and safety glasses. Be careful of drips, spills, and splashes. Keep wet paper towels handy to wipe up any drips. As the activity of the sulfuric acid changes on extended exposure to air, use a uniform mixture of sulfuric acid for the entire assay.

8. Using a repeat pipettor, uniformly add $300 \mu \mathrm{L}$ of $75 \%$ sulfuric acid to each tube. Vortex the tubes vigorously to mix well, pointing the tube away while vortexing. Place the tubes in a heater block at a steady pace in sequential order. Once all the tubes are in, set the timer for 3 min immediately.

9. At $3 \mathrm{~min}$, remove the tubes at a steady pace in the same order, and place them directly in a rack in an ice water bath. Leave the tubes until they are ice cold. Remove the tubes and allow them to equilibrate to room temperature for $\sim 5 \mathrm{~min}$ to prevent condensation on the cuvette during reading.

10. Set a UV/VIS spectrophotometer to read the absorbance at $430 \mathrm{~nm}$ using a $10 \mathrm{~mm}$ pathlength cuvette. Blank with a zero standard tube. Read the absorbance of all the tubes at $430 \mathrm{~nm}$.

NOTE: Disposable plastic cuvettes are convenient to use.

11. Construct a standard curve by plotting $\mu \mathrm{g}$ of carbohydrate standard vs. A430. See Figure 4 for a typical standard curve using glucose as the reference standard.

12. Use the sample assay tubes with $\mathrm{A} 430$ values falling within the linear range of the standard curve, calculate the $\mu \mathrm{g}$ amount of the unknown polysaccharide in the sample assay tubes from the standard curve equation. Determine the concentration of the unknown polysaccharide from the volume of the unknown added, accounting for dilutions. Convert the concentration to $\mathrm{mg} / \mathrm{mL}$ or $\mu \mathrm{M}$ repeat units as required.

3. Hydrazide assay using trinitrobenzene sulfonic acid (TNBS)

1. Prepare $0.9 \% \mathrm{NaCl}$ containing $0.02 \%$ sodium azide (Sample buffer) by dissolving $9 \mathrm{~g}$ of $\mathrm{NaCl}$ and 200 mg of sodium azide in $\mathrm{dl} \mathrm{H}_{2} \mathrm{O}$ to a final volume of $1 \mathrm{~L}$. 
2. Prepare $0.1 \mathrm{M}$ sodium borate, $\mathrm{pH} 9$ (Assay buffer), by mixing $100 \mathrm{~mL}$ of $0.5 \mathrm{M}$ sodium borate, $\mathrm{pH} 9$, with $400 \mathrm{~mL}$ of $\mathrm{dl} \mathrm{H}_{2} \mathrm{O}$. Confirm that the solution $\mathrm{pH}$ is 9 \pm 0.1 ; adjust if necessary.

3. Prepare $1 \%$ TNBS by diluting $200 \mu \mathrm{L}$ of $5 \% 2,4,6$ trinitrobenzene sulfonic acid solution to $1 \mathrm{~mL}$ with $\mathrm{dl}$ $\mathrm{H}_{2} \mathrm{O}$. Mark the tube as $1 \%$ TNBS and store at $4{ }^{\circ} \mathrm{C}$ in the dark for a week.

4. Prepare $50 \mathrm{mM}$ ADH stock (equivalent to $100 \mathrm{mM}$ hydrazide).

1. Weigh out $871 \mathrm{mg}$ of adipic dihyrazide (ADH) powder using an analytical balance. Dissolve the powder in a reagent bottle by adding Sample buffer to $100 \mathrm{~mL}$ with the aid of a top loader balance.

2. Label the bottle as $100 \mathrm{mM}$ hydrazide/50 $\mathrm{mM}$ $\mathrm{ADH}$. Cap the bottle tightly and store at $4{ }^{\circ} \mathrm{C}$ for 1 year.

5. Prepare hydrazide standards $(0.1,0.2,0.3,0.4,0.5$, and $0.6 \mathrm{mM}$ hydrazide).

1. Prepare the 6 hydrazide standards by diluting the $100 \mathrm{mM}$ hydrazide stock with Sample buffer with the aid of a top loader balance. Prepare 100 $\mathrm{mL}$ of each standard to minimize concentration error. Tightly close the bottles and store at $4{ }^{\circ} \mathrm{C}$ for 1 year.

6. Setting up assay reactions

NOTE: The TNBS assay is run at a reaction volume of $1 \mathrm{~mL}$. Each assay tube consists of $100 \mu \mathrm{L}$ of a sample (or a standard), $875 \mu \mathrm{L}$ of Assay buffer, and $25 \mu \mathrm{L}$ of $1 \%$ TNBS solution. All assay reactions (for both samples and standards) are set up in triplicate.
1. Label 3 borosilicate glass tubes $(12 \times 75 \mathrm{~mm})$ for each standard, including the zero standard. Sort and arrange the standard tubes in tube rack in order of increasing concentration. Use a calibrated $100 \mu \mathrm{L}$ or $200 \mu \mathrm{L}$ micropipette to accurately add $100 \mu \mathrm{L}$ of the standards to each corresponding tube. For the zero standard, use $100 \mu \mathrm{L}$ of Sample buffer.

2. Label 3 borosilicate glass tubes $(12 \times 75 \mathrm{~mm})$ for each diluted sample to be assayed. Sort and arrange the sample tubes in the tube rack accordingly. Use a calibrated $100 \mu \mathrm{L}$ or $200 \mu \mathrm{L}$ micropipette to accurately add $100 \mu \mathrm{L}$ of the sample to each corresponding sample tube.

3. Use a calibrated $1000 \mu \mathrm{L}$ micropipette to accurately add $875 \mu \mathrm{L}$ of Assay buffer to all assay tubes: the standards and the samples.

7. To start the assay reaction, use a calibrated $100 \mu \mathrm{L}$ micropipette to accurately add $25 \mu \mathrm{L}$ of $1 \%$ TNBS to each assay tube. Start from the zero standard tubes, move to the standard tubes in order of increasing concentration, then to the sample tubes according to the pre-determined order. Change tips when starting a new standard or a new sample and keep the time spent in adding TNBS to all the tubes to within $5 \mathrm{~min}$.

1. Vortex all assay tubes for $2 \mathrm{~s}$ at high speed or at a speed setting allowing the liquid inside the assay tube to swirl upward to reach a height of $1 / 2$ inch from the tube opening.

2. Record the assay start time and set the timer to $2 \mathrm{~h}$. Place the assay tube rack in the dark at room temperature for $2 \mathrm{~h}$. When the time is over, 
vortex all the tubes one more time and proceed to data collection.

8. Data collection

1. Let the UV/VIS spectrophotometer warm up and the baseline stabilize. Set the detection wavelength at $500 \mathrm{~nm}$ for the hydrazide assay. Use a $1 \mathrm{~mL}$ quartz cuvette of $1 \mathrm{~cm}$ pathlength for all absorbance measurements for the entire assay.

2. Start the data collection by transferring a zero standard assay to the cuvette; blank the instrument (set absorbance to zero).

3. Perform a single read on each tube and record the absorbance values in a data table. Remove any residual liquid from the cuvette before reading a new sample. Start from the zero standards, move to the standards of increasing concentration, and then to the samples. Once started, perform all steps efficiently without stopping and read all tubes within $10 \mathrm{~min}$.

\section{Analyzing sample data}

1. Create a standard curve by plotting $\mathrm{mM}$ hydrazide standard vs. A500. Find the standard curve equation in the form of $y=a x+b$, where $y$ represents $\mathrm{mM}$ hydrazide and $x$ represents A500. See Figure 4 for a typical standard curve.

2. Calculate $\mathrm{mM}$ hydrazide in the samples using the standard curve equation, adjusting for the dilution factors. Choose only the sample assay tubes with $A 500$ values falling within the linear range of the standard curve for the calculation.
3. Calculate the molar ratio of hydrazide/ polysaccharide using equation (1).

Hydrazide/polysaccharide $=h / c \times M W(\mathbf{1})$

Where $h$ is the $\mathrm{mM}$ hydrazide, $c$ is the $\mathrm{mg} / \mathrm{mL}$ concentration of the polysaccharide, and $M W$ is the polysaccharide molecular weight in $\mathrm{kDa}$.

4. Calculate the hydrazide labeling density per 100 $\mathrm{kDa}$ of polysaccharide using equation (2). Labeling density per $100 \mathrm{kDa}$ polysaccharide $=$ $h / c \times 100(2)$

Where $h$ is the $\mathrm{mM}$ hydrazide, and $c$ is the $\mathrm{mg} /$ $\mathrm{mL}$ concentration of the polysaccharide.

NOTE: For convenience, the polysaccharides can be considered to have a molecular weight of 100,000 daltons. This allows one to consider a "labeling density" in comparing the level of derivatization of various polysaccharides.

5. Calculate the hydrazide labeling density as weight percent $A D H$.

1. Determine the effective $\mathrm{mg} / \mathrm{mL}$ concentration of $\mathrm{ADH}$ by using equation (3).

$\mathrm{mg} / \mathrm{mL}$ ADH $=(\mathrm{mM}$ hydrazide $/ 1000) \times 174$ (3)

where 174 is the MW of ADH.

2. Calculate the weight $\% \mathrm{ADH}$ by using equation (4).

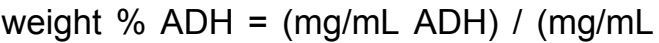
polysaccharide) $\times 100(4)$

\section{Representative Results}

To illustrate the activation and derivatization of a polysaccharide using CDAP chemistry, dextran was activated 
at 0.25 and $0.5 \mathrm{mg} \mathrm{CDAP} / \mathrm{mg}$ dextran. For each reaction, a $10 \mathrm{mg} / \mathrm{mL}$ dextran solution in water was chilled on ice, and $1 / 10^{\text {th }}$ volume of a $2.5 \mathrm{M}$ DMAP stock (prepared as described in section 3) was added. The final solution was brought to $\mathrm{pH}$ 9 by the addition of $0.1 \mathrm{M} \mathrm{NaOH}$ in $10 \mu \mathrm{L}$ aliquots. The solution was chilled and stirred, CDAP added, and the $\mathrm{pH}$ maintained at $\mathrm{pH} 9$ by adding $10 \mu \mathrm{L}$ aliquots of $0.1 \mathrm{M} \mathrm{NaOH}$ for $15 \mathrm{~min}$. Only $0.25 \mathrm{~mL}$ of $0.5 \mathrm{M} \mathrm{ADH}$ at $\mathrm{pH} 9$ was added (less than the usual amount) and the reaction allowed to proceed overnight at $4{ }^{\circ} \mathrm{C}$.

The labeled dextran was then sequentially dialyzed against $1 \mathrm{M} \mathrm{NaCl}, 0.15 \mathrm{M} \mathrm{NaCl}$, and water as described in section 6. The ADH-dextran was then assayed for dextran using the resorcinol/sulfuric acid assay (section 7.2). A typical standard curve using glucose as the sugar standard is shown in Figure 4A. The hydrazide content was determined using the TNBS assay described in section 7.3. A typical hydrazide standard curve using $A D H$ as the standard is given in Figure $4 B$.

Representative calculations from the activation of dextran at the two levels of activation are shown in Figure 4A,B. The data are presented as both hydrazides per $100 \mathrm{kDa}$ of dextran polymer and as a weight percent of $A D H$ to dextran, as described in sections 7.9.3.4 and 7.9.3.5, respectively, in Figure 4C. The degree of derivatization approximately doubled as the CDAP ratio was doubled.

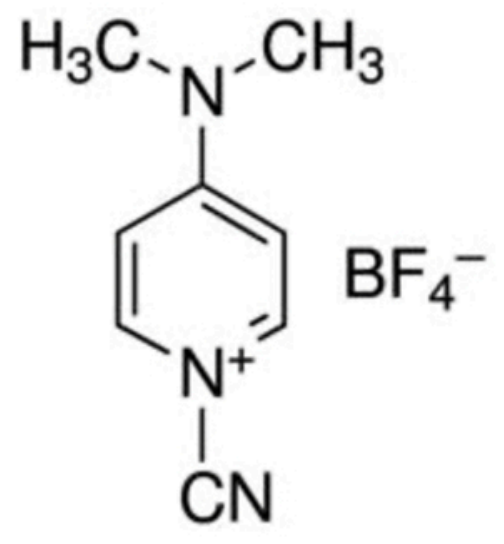

Figure 1: Chemical structure of CDAP. CDAP = 1-cyano-4-dimethylaminopyridine tetrafluoroborate. Please click here to view a larger version of this figure. 


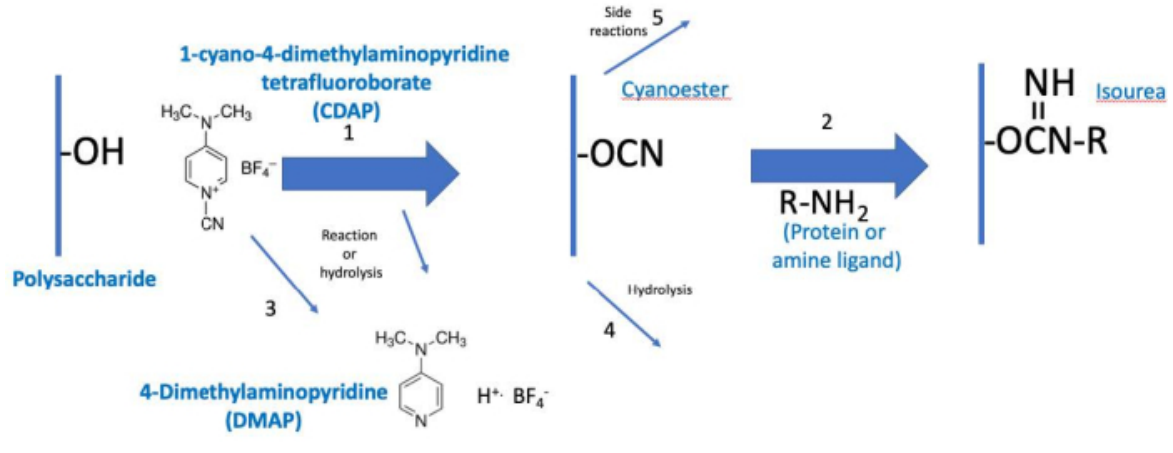

Figure 2: Process of CDAP activation and conjugation. The process is conceptually divided into two phases, with the activated polysaccharide common to both. Under basic conditions, CDAP activates polysaccharide hydroxyls, releasing DMAP (reaction 1). CDAP hydrolysis also releases DMAP (reaction 3). Although a cyano-ester is shown, this may not be the actual intermediate. The intermediate is, therefore, referred to as (CDAP) "activated" polysaccharide. During the first activation phase, the activated polysaccharide can hydrolyze (reaction 4) or undergo side reactions (reaction 5). In the second conjugation phase (reaction 2), the activated polysaccharide reacts with an amine to form a stable isourea bond in addition to reactions 4 and 5. Abbreviations: CDAP = 1-cyano-4-dimethylaminopyridine tetrafluoroborate; DMAP = 4dimethylaminopyridine; $\mathrm{R}-\mathrm{NH}_{2}=$ amine. Please click here to view a larger version of this figure. 
Decreasing $\mathrm{pH}$

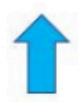

$\downarrow$

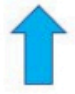

Activated PS stability

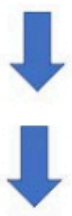

PS reactivity

CDAP stability

\section{Increasing $\mathrm{pH}$}
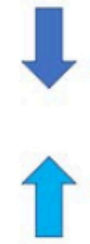

Activated PS

Side reactions

Amine reactivity
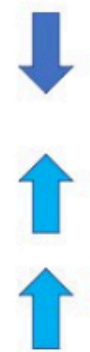

\section{Efficient CDAP activation is a balance between}

1) Reactivity of the polysaccharide and CDAP and

2) Hydrolysis and side reactions of both the reagent and the activated polysaccharide

Figure 3: CDAP activation and conjugation. The process requires balancing CDAP reactivity with the polysaccharide, the stability of the CDAP and activated polysaccharide, as well as the reactivity of the activated polysaccharide with that of the amine. Please click here to view a larger version of this figure. 

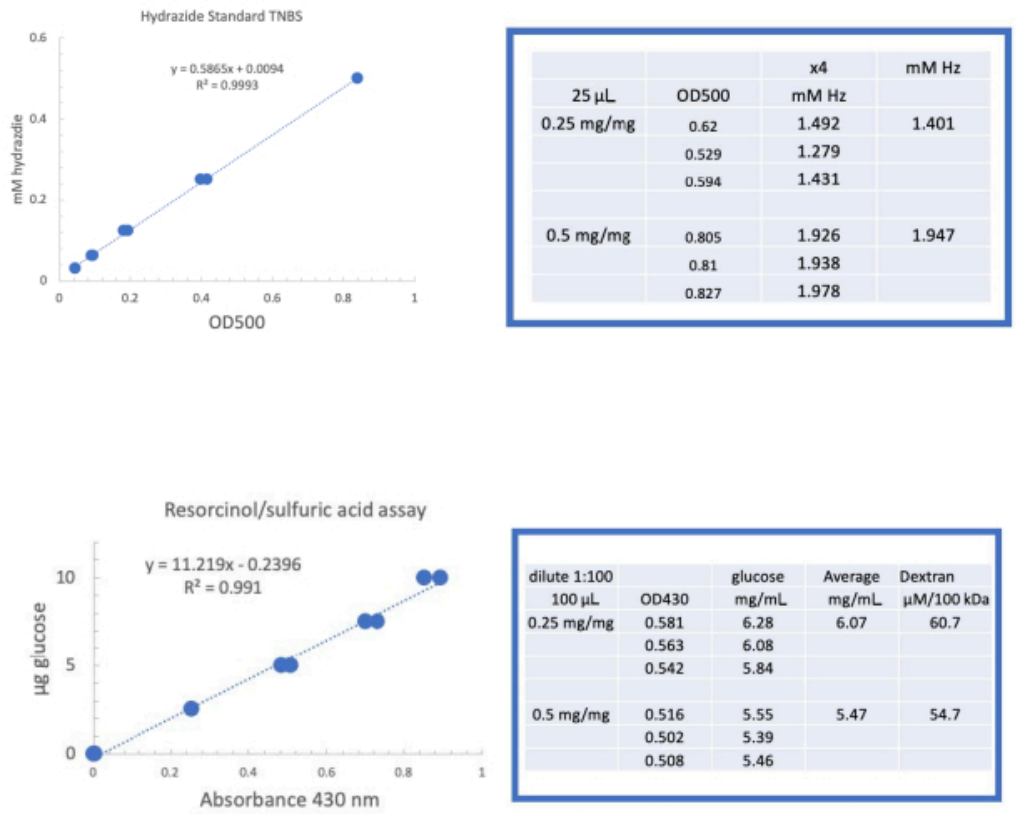

C

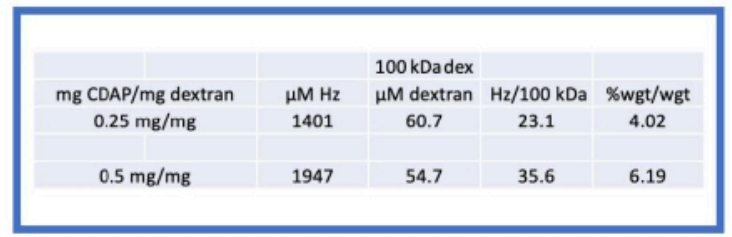

Figure 4: Representative results for CDAP activation of dextran. Typical standard curves for the (A) resorcinol/sulfuric acid and (B) TNBS assays. The assay results for dextran activated with 0.25 and $0.5 \mathrm{mg}$ CDAP/mg dextran are shown. Glucose was used as the standard for the resorcinol assay. Dextran, in $\mathrm{mg} / \mathrm{mL}$, is divided by $100 \mathrm{kDa}$ to give a molar concentration. The hydrazide concentration is determined using $\mathrm{ADH}$ as the standard and the results expressed as $\mu \mathrm{M}$ Hz. (C) Calculation of hydrazide: dextran ratios. The level of derivatization was calculated as hydrazides per $100 \mathrm{kDa}$ of dextran to facilitate the comparison between polymers of different average molecular weights. The $\%$ weight ratio of $g \mathrm{ADH} / \mathrm{g}$ dextran was calculated using a $\mathrm{MW}$ of $174 \mathrm{~g} / \mathrm{mole}$ for $\mathrm{ADH}$. Please click here to view a larger version of this figure.

\section{Discussion}

CDAP is a convenient reagent to derivatize and conjugate polysaccharides. This article describes the general method to use CDAP to derivatize polysaccharides with hydrazides (PS-
$\mathrm{ADH})$ and incorporates recently published improvements ${ }^{8}$.

First, the technique emphasizes the importance of maintaining the target $\mathrm{pH}$ to control the activation process. We found that while many common buffers interfere with 
the CDAP activation reaction, DMAP could successfully be used as the buffer to manage the $\mathrm{pH}^{8}$. Furthermore, DMAP is already a reaction byproduct of CDAP activation. Finally, buffering the polysaccharide solution with DMAP before adding the CDAP facilitates precise targeting and maintenance of the reaction $\mathrm{pH}$. As we describe, it is useful to adjust the $\mathrm{pH}$ of the concentrated DMAP stock solution such that when diluted, it reaches the targeted $\mathrm{pH}$. Secondly, performing the process in the cold slowed the reaction time, making the activation process less frenetic and more forgiving. Lower temperature decreased the rate of CDAP hydrolysis, and the optimal activation time at $\mathrm{pH} 9$ increases from $\sim 3 \mathrm{~min}$ to $\sim 15 \mathrm{~min}$. In addition, less CDAP is required to achieve the same level of activation than when performed at room temperature.

$\mathrm{ADH}$-derivatized polysaccharides can be conjugated to proteins using carbodiimides (e.g., EDAC) ${ }^{7}$. For example, several licensed Haemophilus influenzae b (Hib) vaccines use the polyribosylribitolphosphate (PRP) derivatized with $\mathrm{ADH}$ to conjugate to tetanus toxoid using EDAC. $\mathrm{CNBr}$ was initially employed, but CDAP is a much easier reagent to use for this purpose. In our experience, a good target range for $\mathrm{ADH}$ derivatization is $10-30$ hydrazides per $100 \mathrm{kDa}$ polysaccharide or $\sim 1-3 \%$ ADH by weight.

The same process can be used to derivatize polysaccharides with primary amines by substituting the $A D H$ for a diamine. It is recommended to use hexane diamine to derivatize polysaccharides with amines ${ }^{8}$. The aminated polysaccharide (PS-NH 2 ) can be conjugated using reagents developed for protein conjugation ${ }^{11}$. Typically, the PS$\mathrm{NH}_{2}$ is derivatized with a maleimide (e.g., succinimidyl 4[N-maleimidomethyl]cyclohexane-1-carboxylate (SMCC) or $\mathrm{N}$-y-maleimidobutyryl-oxysuccinimide ester (GMBS)), and the protein is thiolated (e.g., with succinimidyl 3-(2pyridyldithio)propionate (SPDP)). Thiol-maleimide chemistry is very efficient.

Proteins can also be directly coupled to CDAP-activated polysaccharides via the $\varepsilon$-amine on lysines. While the activation protocol used is generally similar to the one described here, it is necessary to optimize the level of activation, polysaccharide and protein concentration, as well as the protein:polysaccharide ratio ${ }^{5,6,8}$.

Dextran is one of the easiest polysaccharides to activate with CDAP due to its relatively high density of hydroxyl groups, but some polysaccharides, such as Vi antigen, can be challenging. Consequently, there is no single "best" protocol for CDAP conjugation directly to proteins. We suggest first developing a protocol to achieve suitable levels of activation, as determined by the extent of hydrazide derivatization, and then proceeding to direct protein conjugation to CDAPactivated polysaccharide.

\section{Disclosures}

Andrew Lees is founder and owner of Fina Biosolutions. He holds several patents relating to CDAP chemistry and benefits from licensing of the chemistry and CDAP conjugation knowhow.

\section{Acknowledgments}

The work described here was funded by Fina Biosolutions LLC.

\section{References}

1. Ellis, R. W.; Granoff, D. M., Development and clinical uses of Haemophilus B conjugate vaccines. CRC Press (1994). 
2. Goebel, W. F., Avery, O. T. Chemo-immunological studies on conjugated carbohydrate-proteins. Journal of Experimental Medicine. 50 (4), 533-550 (1929).

3. Mond, J. J., Vos, Q., Lees, A., Snapper, C. M. T cell independent antigens. Current Opinion in Immunology. 7 (3), 349-354 (1995).

4. Cruse, J. M., Lewis, R. E., Jr. (Eds), Conjugate Vaccines. 10, Karger, Basel (1989).

5. Lees, A., Nelson, B. L., Mond, J. J. Activation of soluble polysaccharides with 1-cyano-4dimethylaminopyridinium tetrafluoroborate for use in protein-polysaccharide conjugate vaccines and immunological reagents. Vaccine. 14 (3), 190-198 (1996).

6. Shafer, D. E. et al. Activation of soluble polysaccharides with 1-cyano-4dimethylaminopyridinium tetrafluoroborate (CDAP) for use in protein-polysaccharide conjugate vaccines and immunological reagents. II. Selective crosslinking of proteins to CDAP-activated polysaccharides. Vaccine. 18 (13), 1273-1281 (2000).

7. Schneerson, R., Barrera, O., Sutton, A., Robbins, J. B. Preparation, characterization, and immunogenicity of Haemophilus influenzae type b polysaccharide-protein conjugates. Journal of Experimental Medicine. 152 (2), 361-376 (1980).

8. Lees, A., Barr, J. F., Gebretnsae, S. Activation of soluble polysaccharides with 1-cyano- 4-dimethylaminopyridine tetrafluoroborate (CDAP) for use in proteinpolysaccharide conjugate vaccines and immunological reagents. III Optimization of CDAP activation. Vaccines. 8 (4), 777 (2020).
9. Qi, X.-Y., Keyhani, N. O., Lee, Y. C. Spectrophotometric determination of hydrazine, hydrazides, and their mixtures with trinitrobenzenesulfonic acid. Analytical biochemistry. 175 (1), 139-144 (1988).

10. Monsigny, M., Petit, C., Roche, A. C. Colorimetric determination of neutral sugars by a resorcinol sulfuric acid micromethod. Analytical biochemistry. 175 (2), $525-530$ (1988).

11. Hermanson, G. Bioconjugate Techniques. 3rd ed., Academic Press (2013). 